\title{
Anti-Proliferative Effects of Evodiamine in Human Lung Cancer Cells
}

\section{Ji-Young Hong, So Hyun Park, Hye-Young Min, Hyen Joo Park, Sang Kook Lee}

College of Pharmacy, Seoul National University, Seoul, Korea

\begin{abstract}
Background: Evodiamine, a compound isolated from the Evodia rutaecarpa Bentham (Rutaceae), is known to have a potential anti-proliferative activity in human cancer cells. However, the growth inhibitory activity against lung cancer cells and the underlying molecular mechanisms have been poorly determined. The present study was designed to examine the anti-proliferative effect of evodiamine in A549 human lung cancer cells.

Methods: A549 cells were treated with the compounds from Evodia rutaecarpa, and the anti-proliferative activity was evaluated by the sulforhodamine B assay. The mechanisms of action for the growth inhibitory activity of evodiamine on A549 human lung cancer cells were evaluated using flow cytometry for cell cycle distribution, and Western blot for assessment of accumulation and phosphorylation of potential target proteins.

Results: Evodiamine exhibited a potent anti-proliferative activity against A549 human lung cancer cells. Flow cytometric analysis revealed that evodiamine induced cell cycle arrest at G2/M phase and apoptosis in the A549 cells. The cell cycle arrest was well correlated with the inhibition of cyclin B1, cyclin A, cdk2 and p-cdc2 (Tyr15) and increase of p-chk1 (Ser345) and p-chk2 (Thr68). Evodiamine also significantly increased the ratio of $\mathrm{Bax} / \mathrm{Bcl}-2$ and decreased procaspase-3, suggesting evodiamineinduced apoptosis via the intrinsic apoptotic pathway. In addition, evodiamine inhibited the expression of p-ERK and ERK. Conclusions: These findings suggest that the anti-proliferative effect of evodiamine was associated in part with the induction of G2/M phase cell cycle arrest and apoptosis, and down-regulation of ERK in human lung cancer cells. (J Cancer Prev 2014;19:7-13)
\end{abstract}

Key Words: Evodiamine, A549 cells, Cell cycle arrest, Apoptosis, Lung cancer

\section{INTRODUCTION}

Lung cancer is one of the most widely diagnosed cancer types in the world and leading cause of cancer-related death. ${ }^{1}$ Despite major advances in development of various targeted therapeutic approaches, mortality related to lung cancer still remains at a high level. For lung cancer therapy, along with surgery, radiotherapy and immunotherapy, chemotherapy is one of the most common treatments. During the past decade, platinum-based combinations with cytotoxic agents such as vinorelbine, gemcitabine and taxanes were used in lung cancer therapy. In recent years, gefitinib and erlotinib have been used clinically for treatment of lung cancer as targeted therapy. However, several side effects and drug resistance are also frequent problems in the course of therapy. ${ }^{2}$ Thus, the development of new effective therapeutic agents to approach lung cancer is extremely necessary.

Evodiamine is a quinazolinecarboline alkaloid isolated from the fruit of Evodia rutaecarpa Bentham. ${ }^{3}$ Evodiamine has been reported to have pharmacological activities of anti-inflammatory, anti-cardiovascular disease, anti-Alzheimer's disease, anti-microbial and anti-obesity. ${ }^{4}$ In addition, evodiamine has anti-cancer effects and inhibits the growth of several types of human cancer cells in vitro and in vivo. ${ }^{5-8}$ In several reports, G2/M phase arrest is believed

Received March 13, 2014, Revised March 18, 2014, Accepted March 18, 2014

Correspondence to: Sang Kook Lee

College of Pharmacy, Seoul National University, San 56-1, Sillim-dong, Gwanak-gu, Seoul 151-742, Korea

Tel: +82-2-880-2475, Fax: +82-2-762-8322, E-mail: sklee61@snu.ac.kr

Copyright (C) 2014 Korean Society of Cancer Prevention

(c) This is an Open Access article distributed under the terms of the Creative Commons Attribution Non-Commercial License (http://creativecommons. $\mathrm{org} /$ licenses/by-nc/3.0) which permits unrestricted non-commercial use, distribution, and reproduction in any medium, provided the original work is properly cited. 
to be one of the major mechanisms of action for evodiamine against human cancer cells. ${ }^{7,9-11}$ However, the mechanisms of evodiamine in the growth-inhibitory activity against human lung cancer cells are poorly defined.

In this study, we examined the anti-proliferative effects of evodiamine in A549 human lung cancer cells. The mechanisms of action of cell cycle arrest and apoptosis were also investigated.

\section{MATERIALS AND METHODS}

\section{Chemicals}

Trichloroacetic acid (TCA), sulforhodamine B (SRB), bovine serum albumin (BSA), propidium iodide (PI), ribonuclease $\mathrm{A}$ (RNase $\mathrm{A}$ ), and anti- $\beta$-actin antibody were purchased from Sigma (St.Louis, MO, USA). RPMI 1640, fetal bovine serum (FBS), trypsin-EDTA and antibioticantimycotic solution were purchased from GIBCO-BRL (Grand Island, NY, USA). Antibodies against Cdk4, Cdk2, Cyclin A, Cyclin B1, Bcl-2, Bax, p-ERK, ERK and CDK1 were purchased from Santa Cruz Biotechnology (Santa Cruz, CA, USA). Antibodies against p-cdc2 (Tyr15), p-Chk1 (Ser345), p-Chk2 (Thr68), Chk1, Chk2, p-H2A.X (Ser139), procaspase-3, caspase-8, LC3B and Beclin-1 were obtained from Cell Signaling (Danvers, MA, USA). The test compounds including evodiamine (Fig. 1) were isolated by Dr. Jung Joon Lee (Korea Research Institute of Bioscience and Biotechnology, Korea) from Evodia rutaecarpa Bentham (Rutaceae) and provided the compounds through the Research Center for Standardization of Herbal Medicines in Korea.

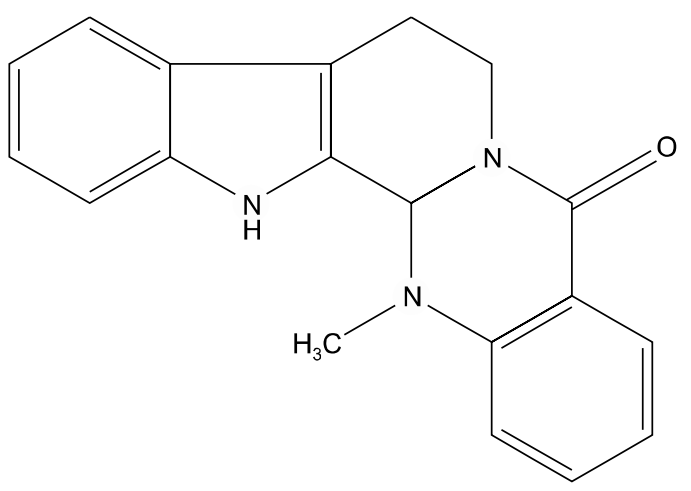

Fig. 1. Chemical structure of evodiamine.

\section{Cell culture}

A549 human lung cancer cells were provided by the Korean Cell Line Bank (Seoul, Korea). The cells were cultured in RPMI1640 medium supplemented with 10\% heat-inactivated fetal bovine serum (FBS) and antibiotics-antimycotics (PSF; 100 units/mL penicillin G sodium, $100 \mu \mathrm{g} / \mathrm{mL}$ streptomycin, and $250 \mathrm{ng} / \mathrm{mL}$ amphotericin B). The cells were incubated at $37^{\circ} \mathrm{C}$ and $5 \% \mathrm{CO}_{2}$ in a humidified atmosphere.

\section{Cell proliferation assay}

Cells $\left(5 \times 10^{4}\right.$ cells $\left./ \mathrm{ml}\right)$ were treated with various concentrations of compounds (total volume of $200 \mu \mathrm{l} /$ well) in 96-well culture plates for $72 \mathrm{~h}$. After treatment, the cells were fixed with 10\% TCA solution, and cell viability was determined with a sulforhodamine B (SRB) assay. ${ }^{12}$ The results were expressed as percentages relative to solvent-treated control incubations, and $\mathrm{IC}_{50}$ values were calculated using non- linear regression analysis (percent survival versus concentration).

\section{Cell cycle analysis}

A549 cells were plated at a density of $1 \times 10^{6}$ cells per 100 $\mathrm{mm}$ culture dish and incubated for $24 \mathrm{~h}$. Fresh media containing various concentrations of test sample were added to culture dishes. Following a $24 \mathrm{~h}$ incubation, the cells were harvested (trypsinization and centrifugation) and fixed with $70 \%$ ethanol overnight at $4^{\circ} \mathrm{C}$. Fixed cells were washed with PBS and incubated with a staining solution containing RNase A $(50 \mu \mathrm{g} / \mathrm{ml})$ and propidium iodide $(50 \mu \mathrm{g} / \mathrm{ml})$ in PBS for $30 \mathrm{~min}$ at room temperature. The cellular DNA content was analyzed with a FACSCalibur_ flow cytometer (BD Biosciences, San Jose, CA, USA). Approximately 20,000 cells were used for each analysis, and the distribution of cells in each phase of the cell cycle was displayed as histograms.

\section{Western blot analysis}

A549 human lung cancer cells were exposed with various concentrations of evodiamine for $24 \mathrm{~h}$. After incubation, cells were lysed and protein concentrations were determined by BCA method. Each protein (40 $\mu \mathrm{g}$ ) was subjec- 
ted to SDS-PAGE. Proteins were transferred onto PVDF membranes by electroblotting, and membranes were treated for $1 \mathrm{~h}$ with blocking buffer [5\% non-fat dry milk in phosphate-buffered saline-0.1\% Tween 20 (PBST)]. Membranes were then incubated with indicated antibodies (mouse anti- $\beta$-actin, diluted 1:2,000; other antibodies, diluted 1:1,000 in PBST) overnight at $4{ }^{\circ} \mathrm{C}$, washed three times for 5 min with PBST. After washing, membranes were incubated with HRP-conjugated anti-mouse IgG diluted 1 : 2,000 in PBST for $2 \mathrm{~h}$ at room temperature, washed three times for 5 min with PBST, and visualized by HRP-chemiluminescent detection kit (Lab Frontier, Seoul, Korea) using LAS-3000 Imager (Fuji Film Corp., Japan).

\section{Statistical analysis}

All of the experiments were repeated at least three times. Data were expressed as the mean \pm standard deviation (SD) for the indicated number of independently performed experiments and analyzed using Student's t-test. Values of $\mathrm{P}<0.05$ were considered statistically significant.

\section{RESULTS}

\section{Anti-proliferative effects of evodiamine on A549 human lung cancer cells}

A549 human lung cancer cells were treated with several compounds isolated from Evodia rutaecarpa for $72 \mathrm{~h}$, and the cell proliferation of each tested group was determined by the SRB staining method. As shown in Table 1, among the test compounds, evodiamine exhibited the most potent anti-proliferative effects. Evodiamine exhibited a remar-

Table 1. Inhibitory effects of compunds from Evodia rutaecarpa on the proliferation of A549 human lung cancer cells. Cells were treated with various concentrations of compounds for $72 \mathrm{~h}$. Cell viability was determined by the SRB assay

\begin{tabular}{lc}
\hline \multicolumn{1}{c}{ Compounds } & $\mathrm{IC}_{50}(\mu \mathrm{M})$ \\
\hline Evodiamine & 1.3 \\
Rutaecarpine & $>100$ \\
Goshuyuamide $\|$ & $>100$ \\
Limonin & $>100$ \\
Rutaevine & $>100$ \\
Evodol $_{\text {Ellipticine }}^{\mathrm{a}}$ & $>100$ \\
\hline
\end{tabular}

${ }^{a}$ Ellipticine was used as a positive control. kable anti-proliferative activity with the $\mathrm{IC}_{50}$ value of $1.3 \mu \mathrm{M}$ (Fig. 2). The $\mathrm{IC}_{50}$ value of ellipticine, a positive control, was $0.8 \mu \mathrm{M}$ in the A549 cells under the same experimental condition.

\section{Effects of evodiamine on cell cycle distribution}

To investigate the anti-proliferative mechanisms of evodiamine, A549 cells were treated with the indicated concentration of evodiamine for $24 \mathrm{~h}$, and flow cytometric analysis was performed with propidium iodide (PI) staining. As shown in Fig. 3, the distribution (\%) of cells in the G2/M phase was markedly increased by evodiamine in a concentration-dependent manner. In addition, the treatment of evodiamine slightly increased the cell cycle distribution in the sub-G1 phase, indicative of apoptotic cell death.

\section{Effects of evodiamine on the expression of $\mathrm{G} 2 / \mathrm{M}$ phase cell cycle checkpoint proteins}

To further elucidate whether cell cycle arrest is associated with the regulation of cell cycle checkpoint proteins, the expression of G2/M cell cycle regulatory proteins was examined by Western blot analysis. As shown in Fig. 4, the expression of cyclin A, cyclin B1, cdk2 and p-cdc2

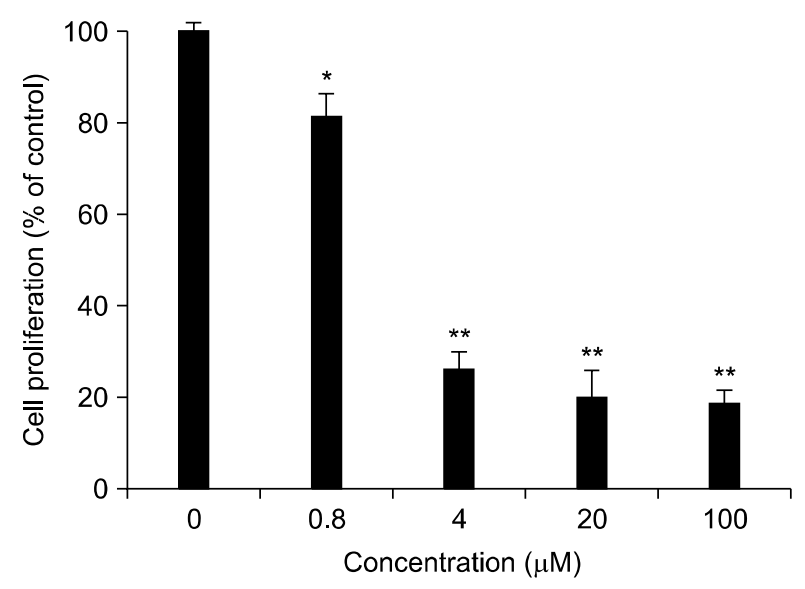

Fig. 2. Effect of evodiamine on the proliferation of A549 human lung cancer cells. A549 cells were plated at 10,000 cells in 96-well plate in RPMI supplemented with $10 \%$ FBS, and incubated with the test compound as the indicated concentrations for $72 \mathrm{~h}$. Anti-proliferative effect was determined using SRB assay. The values of \% cell growth are calculated by the mean absorbance of samples/absorbance of vehicletreated control. Data are represented as the means \pm S.D. $(n=3)$. 
Cell cycle distribution (\%)
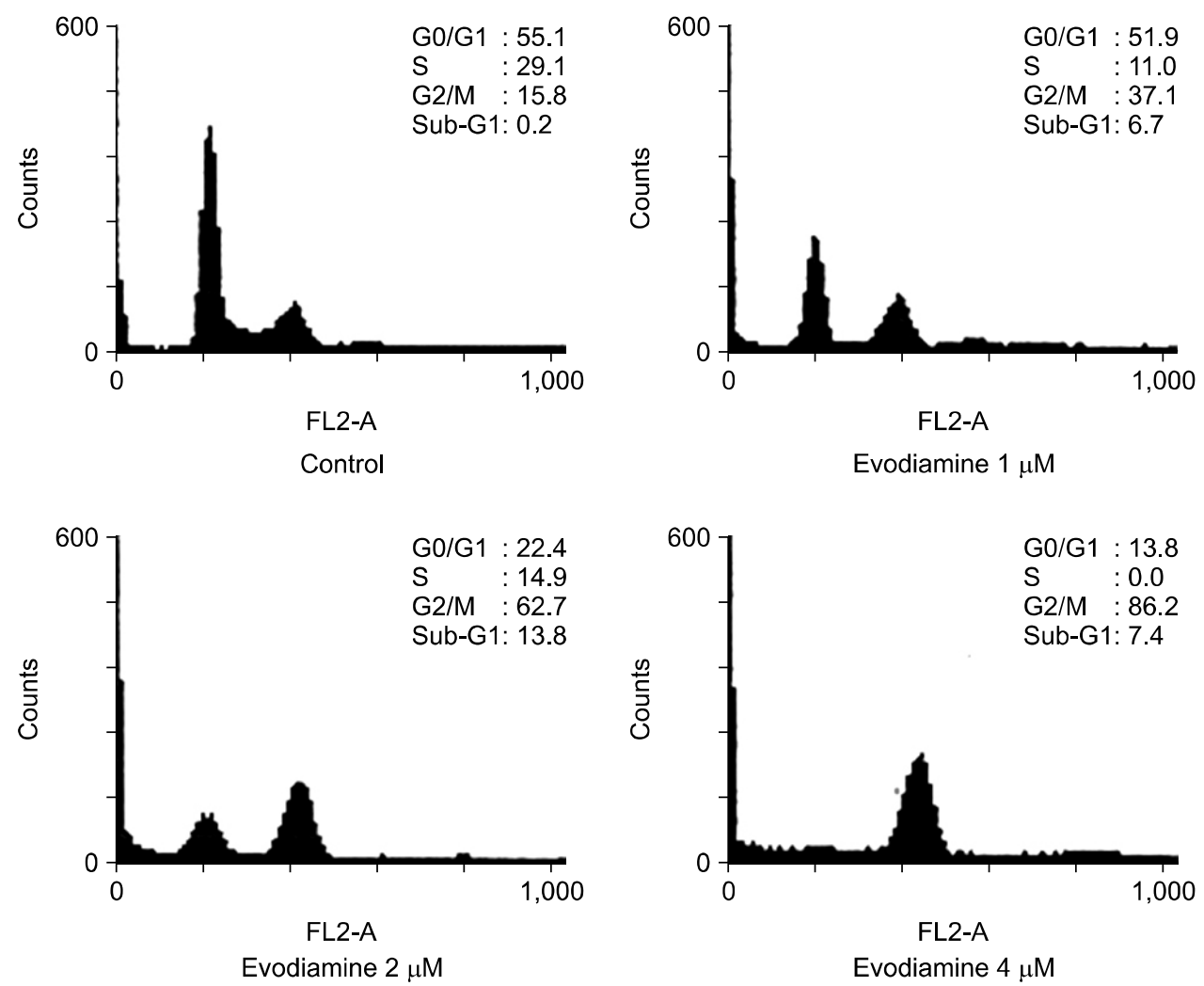

Fig. 3. Effect of evodiamine on the regulation of cell cycle distribution in A549 cells. Cells were seeded at $1 \times 10^{6}$ cells in $100 \mathrm{~mm}$ dish in RPMI supplemented with $10 \% \mathrm{FBS}$, and then treated with various concentration of evodiamine for 24 h. The cell cycle distribution was analyzed by flow cytometry as described in Materials and Methods.

(Tyr15) was significantly decreased and the levels of p-chk1 (Ser345), p-chk2 (Thr68) and p-H2A.X (Ser139) were increased. Evodiamine treatment did not affect the levels of cdc2.

\section{Effects of evodiamine on the apoptosis- and auto- phagy-related proteins}

The changes in the expression of apoptosis-inducing signaling molecules were evaluated in evodiamine-treated A549 cells. Upon treatment with evodiamine for $24 \mathrm{~h}$, the level of Bax was increased while Bcl-2 and procaspase-3 levels were decreased in A549 cells. However, the cleaved form of caspase-8 did not appeared (Fig. 5A). In addition, the levels of LC3B-II and Beclin-1, autophagy markers were elevated by evodiamine treatment (Fig. 5B). Furthermore, evodiamine decreased the expression of p-ERK and ERK (Fig. 5C).

\section{DISCUSSION}

Although many reports have elucidated the anti-proliferative activities of evodiamine in a variety of cancer cells, little is known about the mechanism of evodiamine-induced anti-proliferative activity in human lung cancer cells. This study was designed to evaluate the effect of evodiamine on the proliferation of human lung cancer cells and thereof further investigate the mechanisms of action.

Initially, the anti-proliferative activities of isolates from the fruits of Evodia rutaecarpa in A549 human lung cancer cells were examined. Among the compounds tested, only evodiamine exhibited potent anti-proliferative activity with the $\mathrm{IC}_{50}$ value of $1.3 \mu \mathrm{M}$. Based on the potential anti-proliferative effect of evodiamine, we further investigated its mechanisms of action in terms of cell cycle arrest and regulation of cell-cycle regulatory proteins in A549 cells. As shown in Fig. 3, over $2 \mu \mathrm{M}$ of evodiamine drastically increased the cell cycle distribution of $\mathrm{G} 2 / \mathrm{M}$ 
phase compared with control cells. This result is consistent with previous reported data on G2/M phase arrest by evodiamine. ${ }^{7,10,11,13}$ Cyclin B1 is a key component of the G2

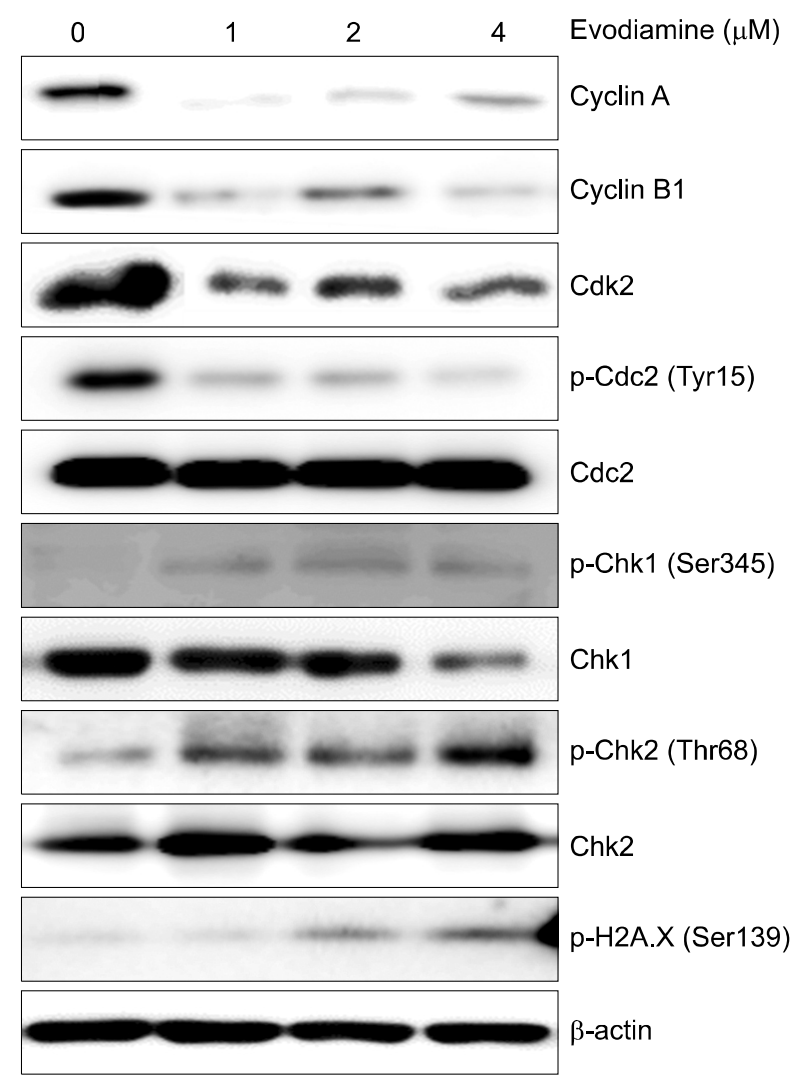

Fig. 4. Effect of evodiamine on the expression of cell cycle associated proteins in A549 cells. Cells were treated with the indicated concentrations of evodiamine for $24 \mathrm{~h}$. The expression level of proteins was analyzed by Western blot.

A

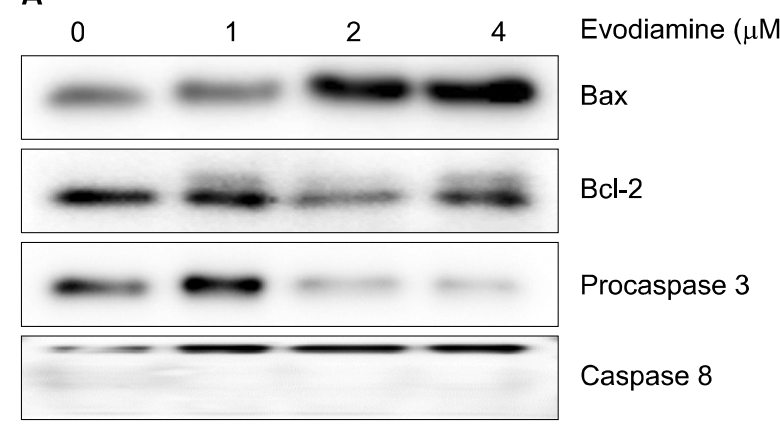

to $\mathrm{M}$ phase transition. ${ }^{14}$ In addition, cyclinA/Cdk2 complexes play a role in the initiation of mitosis in human cancer cells. ${ }^{15}$ In this study, we found that the induction of cell cycle arrest at G2/M phase by evodiamine is in part mediated through the down-regulation of cyclin A, cyclin B1, cdk2 and p-cdc2 (Tyr15). In order to further elucidate the molecular mechanism underlying evodiamine-induced G2/M phase arrest, we investigated the activation of the DNA damage signaling pathway. DNA damage sensor Chk1/Chk2 plays a role in G2/M checkpoint via the ataxia-telangiectasia mutated (ATM)/ATM-RAD3-related (ATR) pathway. Treatment of evodiamine increased the phosphorylation of Chk1 and Chk2 (Fig. 4). In addition, the phosphorylation of H2A.X, which is the molecular maker of DNA double-strand breaks, was increased by evodiamine. Based on these observations, it is likely that the anti-proliferative mechanism of evodiamine is related to the induction of G2/M phase cell cycle arrest by inhibiting the levels of cyclin B1 and Cdc-2 (Tyr15) and increase of Chk1 and Chk2 signaling pathway.

Apoptosis is an energy-dependent programmed cell death that is crucial to the balance between cellular survey and death. ${ }^{16}$ In the mitochondria-dependent pathway (intrinsic pathway), the increased pro-apoptotic members of Bcl-2 family of protein, such as Bax, induce the release of cytochrome c into the cytosol via the opening of the outer mitochondrial membrane. ${ }^{17}$ The caspase activation pathway (extrinsic apoptotic pathway) involves the triggering of the death-receptor superfamily, such as the TNF

\section{B}

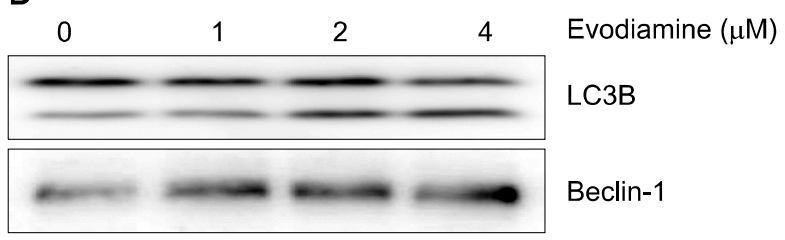

C

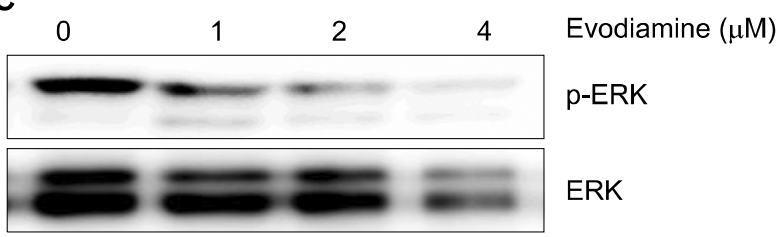

Fig. 5. Effects of evodiamine on the expression of apoptosis and autophagy associated proteins. A549 cells were treated for 24 $\mathrm{h}$ with various concentrations of evodiamine and the protein expressions were measured by Western blot analysis. 
receptor-1 or Fas receptor with its ligand. Numerous studies have indicated that evodiamine induces apoptosis against human cancer cells. ${ }^{17}$ Therefore, we further examined the expression of the apoptosis-related proteins such as Bcl-2, Bax, caspase-3 and caspase-8 in A549 human lung cancer cells. As shown in Fig. 5A, evodiamine suppressed the expression of Bcl-2, Bax and caspase-3. However, evodiamine did not affect the cleavage of caspase-8, the marker of extrinsic apoptotic pathway. In a previous study, evodiamine elevated the activities of caspase-8 in most cancer cells such as DU145 prostate cancer, SGC-7901 gastric cancer and 253J and T24 bladder cancer cells, however not in PC-3 prostate cancer cells similar to the results of our present study. ${ }^{13,18,19}$ This difference in regulation of caspase- 8 by evodiamine seems to be different genetic background or biologically diverse characteristics in cells. Overall, these results suggest that the induction of apoptosis by evodiamine is more likely to be associated with the intrinsic apoptotic pathway rather than the extrinsic apoptotic pathway in A549 cells.

Autophagy is a cellular catabolic degradation response to starvation or stress whereby cellular proteins, organelles and cytoplasm are engulfed, digested and recycled to sustain cellular metabolism. ${ }^{20,21}$ In previous reports, evodiamine induced autophagy and apoptosis in U87-MG glioblastoma and SGC-7901 gastric cancer cells. ${ }^{22,23}$ In U87-MG cells, autophagy and apoptosis were accompanied by increased cytoplasmic accumulation of calcium. ${ }^{22}$ Rasul et al. have demonstrated that beclin-1 is involved in evodiamine-induced autophagy in SGC-7901 cells. ${ }^{23}$ In the present study, two bands, LC3-I (up) and LC3-II (down), were detected by Western blot analysis of proteins from evodiamine-treated cells. Therefore, the modification of LC3-I to LC3-II which is correlated with the extent of autophagosome formation was observed with treatment of evodiamine. Furthermore, beclin-1, autophagy regulation protein, was increased by evodiamine. Recently, it has been reported that natural products, such as plumbagin, oridonin and curcumin induced beclin 1-dependent autophagic cell death. ${ }^{24-26}$ Further studies, therefore, are warranted to determine whether evodiamine induces autophagic vacuoles.

The mitogen-activated protein kinase (MAPK) pathways modulate gene expression, differentiation, cell cycle arrest, mitosis, proliferation, motility, metabolism and apoptosis. ${ }^{27,28}$ The MAPK signaling pathways are generally subdivided into three separate pathways: ERK, p38 MAPK and JNK pathways. Many studies have suggested that the MAPK signaling pathway is central to the growth and survival of NSCLC. ${ }^{29}$ To determine the specific pathway involved in evodiamine-induced cell death, the effects of evodiamine on MAPK activation were also examined. The findings demonstrate that evodiamine inhibited phosphorylation and expression of ERK, but had no effect on either p38 MAPK or JNK1/2 (data not shown), suggesting that the activation of $\mathrm{p} 38$ and JNK1/2 does not play a critical role in evodiamine-induced apoptosis.

In conclusion, the anti-proliferative effects of evodiamine in A549 cells were in part associated with cell cycle arrest at the G2/M phase and induction of apoptosis. These results suggest that evodiamine is an effective natural compound for the treatment of lung cancer.

\section{ACKNOWLEDGEMENTS}

This research was supported by a grant (2005 and 12172MFDS989) from Ministry of Food and Drug Safety in 2014.

\section{REFERENCES}

1. Siegel R, Naishadham D, Jemal A. Cancer statistics, 2012. CA: a Cancer Journal for Clinicians 2012;62:10-29.

2. Reungwetwattana T, Dy GK. Targeted therapies in development for non-small cell lung cancer. Journal of Carcinogenesis 2013;12:22-65.

3. King CL, Kong YC, Wong NS, Yeung HW, Fong HH, Sankawa U. Uterotonic effect of Evodiaminedia rutascarpa alkaloids. Journal of Natural Products 1980;43:577-82.

4. Yu H, Jin H, Gong W, Wang Z, Liang H. Pharmacological actions of multi-target-directed evodiamine. Molecules 2013; 18:1826-43.

5. Du J, Wang XF, Zhou QM, Zhang TL, Lu YY, Zhang H et al. Evodiamine induces apoptosis and inhibits metastasis in MDA-MB-231 human breast cancer cells in vitro and in vivo. Oncology Reports 2013;30:685-94.

6. Chan ALF, Chang WS, Chen LM, Lee CM, Chen CE, Lin $\mathrm{CM}$ et al. Evodiamine stabilizes topoisomerase I-DMA cleavable complex to inhibit topoisomerase I activity. Molecules 2009;14:1342-52.

7. Huang DM, Guh JH, Huang YT, Chueh SC, Chiang PC, 
Teng CM. Induction of mitotic arrest and apoptosis in human prostate cancer $\mathrm{PC}-3$ cells by evodiamine. The Journal of Urology 2005;173:256-61.

8. Zhang Y, Wu LJ, Tashiro S, Onodera S, Ikejima T. Intracellular regulation of evodiamine-induced A375-S2 cell death. Biological \& Pharmaceutical Bulletin 2003;26:1543-7.

9. Yang Y, Zhu X, Chen Y, Wang X, Chen R. p38 and JNK MAPK, but not ERK1.2 MAPK, play important role in colchicines-induced cortical neurons apoptosis. European Journal of Pharmacolgy 2007;576:26-33.

10. Kan SF, Huang WJ, Lin LC, Wang PS. Inhibitory effects of evodiamine on the growth of human prostate cancer cell line LNCaP. International Journal of Cancer 2004;110: 641-51.

11. Chen MC, Yu CH, Wang SW, Pu HF, Kan SF, Lin LC et al. Anti-proliferative effects of evodiamine on human thyroid cancer cell line ARO. Journal of Cellular Biochemistry 2010;110:1495-1503.

12. Hong JY, Chung HJ, Lee HJ, Park HJ, Lee SK. Growth inhibition of human lung cancer cells via down-regulation of epidermal growth factor receptor signaling by yuanhuadine, a daphnane diterpene from Daphne genkwa. Journal of Natural Products 2011;74:2102-8.

13. Yang L, Liu X, Wu D, Zhang M, Ran G, Bi $Y$ et al. Growth inhibition and induction of apoptosis in SGC-7901 human gastric cancer cells by evodiamine. Molecular medicine reports 2014;9:1147-52.

14. Jackman M, Lindon C, Nigg EA, Pines J. Active cyclin B1-Cdk1 first appears on centrosomes in prophase. Nat Cell Biology 2003;5:143-8.

15. Mitra J, Enders GH. Cyclin A/Cdk2 complexes regulate activation of Cdk1 and Cdc25 phosphatases in human cancer cells. Oncogene 2004;23:3361-7.

16. Earnshaw WC. Apoptosis. A cellular poison cupboard. Nature 1999;397:387-9.

17. Zimmermann KC, Bonzon C, Green DR. The machinery of programmed cell death. Pharmacology \& Therapeutics 2001; 92:57-70.

18. Anti-proliferative effects of evodiamine on human prostate cancer cell lines DU145 and PC3. Journal of Cellular Biology 2007;101:44-56.

19. Zhang T, Qu S, Shi Q, He D, Jin X. Evodiamine induces apoptosis and enhances TRAIL-induced apoptosis in human bladder cancer cells through mTOR/S6K1-mediated downregulation of $\mathrm{Mcl}-1$. International Journal of Molecular Sciences 2014;15:3154-71.

20. Kamada Y, Sekito T, Ohsumi Y. Autophagy in yeast: a TOR-mediated response to nutrient starvation. Current Topics in Microbiology and Immunology 2004;279:73-84.

21. Levine B, Klionsky DJ. Development by self-digestion: molecular mechanisms and biological functions of autophagy. Developmental Cell 2004;6:463-77.

22. Liu AJ, Wang SH, Chen KC, Kuei HP, Shih YL, Hou SY et al. Evodiamine, a plant alkaloid, induces calcium/JNK-mediated autophagy and calcium/mitochondria-mediated apoptosis in human glioblastoma. Chemico-Biological Interactions 2013;205:20-8.

23. Rasul A, Yu B, Zhong L, Khan M, Yang H, Ma T. Cytotoxic effect of evodiamine in SGC-7901 human gastric adenocarcinoma cells via simultaneous induction of apoptosis and autophagy. Oncology Reports 2012;27:1481-7.

24. Aoki H, Takada Y, Kondo S, Sawaya R, Aggarwal BB, Kondo Y. Evidence that curcumin suppresses the growth of malignant gliomas in vitro and in vivo through induction of autophagy: role of akt and extracellular signal-regulated kinase signaling pathways. Molecular Pharmacology 2007;72:29-39.

25. Kuo PL, Hsu YL, Cho CY. Plumbagin induces G2/M arrest and autophagy by inhibiting the AKT/mammalian target of rapamycin pathway in breast cancer cells. Molecular Cancer Therapeutics 2006;5:3209-21.

26. Li D, Cui Q, Chen SG, Wu LJ, Tashiro S, Onodera S, Ikejima T. Inactivation of ras and changes of mitochondrial membrane potential contribute to oridonin-induced autophagy in a 431 cells. Journal of Pharmacological Sciences. 2007;105:22-33.

27. Wada T, Penninger JM. Mitogen-activated protein kinases in apoptosis regulation. Oncogene 2004;23:2838-49.

28. Keyse SM. Dual-specificity MAP kinase phosphatases (MKPs) and cancer. Cancer Metastasis Reviews 2008;27:253-61.

29. Marinov M, Fischer B, Arcaro A. Targeting mTOR signaling in lung cancer. Critical Reviews in Oncology/Hematology 2007;63:172-82. 